\title{
Die operative Behandlung der Azetabulum-T-Fraktur über eine chirurgische Hüftluxation oder einen Stoppa-Zugang
}

\author{
Moritz Tannast, Klaus-Arno Siebenrock
}

\section{Zusammenfassung Operationsziel}

Anatomische Reposition und stabile Osteosynthese mit weichteilschonenden Zugängen.

\section{Indikationen}

Dislozierte Azetabulum-T-Frakturen.

Chirurgische Hüftluxation: Bei größerer Dislokation des hinteren Pfeilers im Vergleich zum vorderen Pfeiler, transtektalen Frakturen, zusätzlichen intraartikulären Fragmenten, Gelenkimpaktion.

Stoppa-Zugang: Bei größerer Dislokation des vorderen Pfeilers im Vergleich zum hinteren Pfeiler.

Bei schwieriger Reposition sind u.U. zwei Zugänge notwendig.

\section{Kontraindikationen}

Alter der Frakturen > 15 Tage (dann eher klassischer ilioinguinaler oder erweiterter iliofemoraler Zugang). Stoppa-Zugang: Suprapubischer Blasenkatheter und abdominale Problematik, z.B. nach Laparotomie aufgrund viszeraler Verletzungen (dann eher klassischer ilioinguinaler Zugang).

\section{Operationstechnik}

Chirurgische Hüftluxation: Seitenlage. Gerade laterale Inzision über Trochanter major und Spalten des Gibson-Intervalls. Digastrische Trochanterosteotomie unter Schonung der Arteria circumflexa femoris medialis. Eröffnung des Intervalls zwischen Musculus piriformis und Musculus gluteus minimus. Z-förmige Kapsulotomie. Luxation des Femurkopfes. Reposition des hinteren Pfeilers und Osteosynthese mit einer Rekonstruktionsplatte über dem dorsalen Pfeiler. Reposition des vorderen Pfeilers und Fixation mit Zugschraube in Richtung des oberen Schambeinasts. Stoppa-Zugang: Rückenlage. Pfannenstiel-Inzision, Längsspalten der Rektusscheide. Spalten des Musculus rectus abdominis. Stumpfe Eröffnung des Retzius-Raums. Ligatur einer allfälligen Corona mortis. Stumpfe Präparation der quadrilateralen Fläche und des vorderen Pfeilers. Reposi- tion des vorderen Pfeilers und Fixation mit einer Rekonstruktionsplatte. Fixation des hinteren Pfeilers über Schrauben. Falls notwendig, zusätzliche Eröffnung des ersten Fensters des klassischen ilioinguinalen Zugangs.

\section{Weiterbehandlung} Während der Hospitalisation regelmäßige Behandlung auf der passiven Bewegungsschiene mit maximal $90^{\circ} \mathrm{Fle}-$ xion. Nach chirurgischer Hüftluxation keine aktive Abduktion, keine passive Adduktion über die Mittellinie mit Überkreuzen der Beine, kein Heben des gestreckten Beins. 10-15 kg Teilbelastung an zwei Unterarmgehstöcken während 8 Wochen. Anschließend erste klinische und radiologische Nachkontrolle und je nach Befund schrittweiser Übergang zur Vollbelastung. Thromboseprophylaxe bis zur Vollbelastung.

\section{Ergebnisse}

17 Patienten mit einem mittleren Nachuntersuchungszeitraum von 3,2 Jahren. Zehn Patienten wurden via chirurgische Hüftluxation, zwei über Stoppa-Zugang und fünf über einen kombinierten oder alternativen Zugang operiert. Anatomische Reposition gemäß Matta-Kriterien bei zehn der zwölf Patienten (83\%) ohne primäre endoprothetische Versorgung. Mittlere Operationszeit 3,3 h für chirurgische Hüftluxation und 4,2 h für Stoppa-Zugang. Komplikationen umfassten eine verzögerte Trochanterheilung, heterotope Ossifikationen Grad II-III nach Brooker bei einem Patienten sowie einen sekundären Repositionsverlust. Es wurden keine Hüftkopfnekrosen beobachtet. Bei zwei Patienten musste im Verlauf trotz anatomischer Rekonstruktion des Gelenks aufgrund einer sekundären Koxarthrose eine Hüfttotalprothese implantiert werden.

\section{Schlüsselwörter}

Azetabulumfraktur · T-Fraktur · Chirurgische Hüftluxation · Stoppa-Zugang

\author{
Oper Orthop Traumatol 2009;21:251-69 \\ DOI 10.1007/s00064-009-1803-7
}

${ }^{1}$ Klinik und Poliklinik für Orthopädische Chirurgie, Inselspital, Universität Bern, Schweiz. 


\section{Operative Treatment of T-Type Fractures of the Acetabulum via Surgical Hip Dislocation or Stoppa Approach}

\section{Abstract \\ Objective}

Anatomic reduction and stable fixation by means of tissue-preserving surgical approaches.

\section{Indications}

Displaced acetabular fractures.

Surgical hip dislocation approach with larger displacement of the posterior column in comparison to the anterior column, transtectal fractures, additional intraarticular fragments, marginal impaction.

Stoppa approach with larger displacement of the anterior column in comparison to the posterior column.

A combined approach might be necessary with difficult reduction.

\section{Contraindications}

Fractures > 15 days (then ilioinguinal or extended iliofemoral approaches).

Suprapubic catheters and abdominal problems (e.g., previous laparotomy due to visceral injuries) with Stoppa approach (then switch to classic ilioinguinal approach).

\section{Surgical Technique}

Surgical hip dislocation: lateral decubitus position. Straight lateral incision centered over the greater trochanter. Entering of the Gibson interval. Digastric trochanteric osteotomy with protection of the medial circumflex femoral artery. Opening of the interval between the piriformis and the gluteus minimus muscle. Z-shaped capsulotomy. Dislocation of the femoral head. Reduction and fixation of the posterior column with plate and screws. Fixation of the anterior column with a lag screw in direction of the superior pubic ramus.

Stoppa approach: supine position. Incision according to Pfannenstiel. Longitudinal splitting of the anterior portion

\section{Vorbemerkungen}

Eine T-Fraktur des Azetabulums findet sich in etwa 3-12\% aller Azetabulumfrakturen. Sie ist definiert als eine transversale Azetabulumfraktur in Verbindung mit einem vertikalen Frakturspalt, welcher den vorderen vom hinteren Pfeiler separiert. Obwohl beide Pfeiler frakturiert sind, unterscheidet sich die T-Fraktur per definitionem von einer Zwei-Pfeiler-Fraktur dadurch, dass noch ein Teil der azetabulären Gelenkfläche im stabilen Verbund mit dem iliakalen Beckenring steht. Abhängig von der Höhe der transversalen Frakturlinie of the rectus sheet and the rectus abdominis muscle. Blunt dissection of the space of Retzius. Ligation of the corona mortis, if present. Blunt dissection of the quadrilateral plate and the anterior column. Reduction of the anterior column and fixation with a reconstruction plate. Fixation of the posterior column with lag screws. If necessary, the first window of the ilioinguinal approach can be used for reduction and fixation of the posterior column.

\section{Postoperative Management} During hospital stay, intensive mobilization of the hip joint using a continuous passive motion machine with a maximum flexion of $90^{\circ}$. No active abduction and passive adduction over the body's midline, if a surgical dislocation was performed. Maximum weight bearing 10-15 kg for 8 weeks. Then, first clinical and radiographic follow-up. Deep venous thrombosis prophylaxis for 8 weeks postoperatively.

\section{Results}

17 patients with a mean follow-up of 3.2 years. Ten patients were operated via surgical hip dislocation, two patients with a Stoppa approach, and five using a combined or alternative approach. Anatomic reduction was achieved in ten of the twelve patients (83\%) without primary total hip arthroplasty. Mean operation time $3.3 \mathrm{~h}$ for surgical hip dislocation and $4.2 \mathrm{~h}$ for the Stoppa approach.

Complications comprised one delayed trochanteric union, one heterotopic ossification, and one loss of reduction. There were no cases of avascular necrosis. In two patients, a total hip arthroplasty was performed due to the development of secondary hip osteoarthritis.

\section{Key Words}

Acetabular fracture - T-type · Surgical hip dislocation - Stoppa approach unterscheidet man eine transtektale (ca. 27\%), eine juxtatektale (ca. 45\%) und eine infratektale (ca. 28\%) Fraktur (Abbildung 1a). Der vertikale Spaltbruch (Abbildung 1b) läuft in den meisten Fällen durch das Foramen obturatum (ca. 62\%), selten anterior durch den Ramus inferior ossis pubis (ca. 18\%) oder posterior durch das Tuber ischiadicum (ca. 20\%), wobei dieser Anteil der Fraktur auch inkomplett sein kann.

Konventionell radiologisch zeigt sich in der Beckenübersichtsaufnahme (Abbildungen 2a bis 2c) eine Unterbrechung der ilioischialen und iliopektinealen Linie 


\section{Abbildungen 1a und $\mathbf{1 b}$}

a) Klassifikation der transversalen Frakturkomponente der Azetabulum-T-Fraktur. Die transtektale Fraktur verläuft durch die Facies lunata des Azetabulumsdachs. Die juxtatektale Fraktur verläuft durch den kranialen Anteil der Fossa acetabuli. Die infratektale Fraktur schneidet den inferioren Anteil des Vorder- und Hinterhorns.

b) Je nach Richtung des T-Stiels wird eine anteriore, vertikale und posteriore T-Fraktur unterschieden. Dabei können die Frakturen auch inkomplett sein.
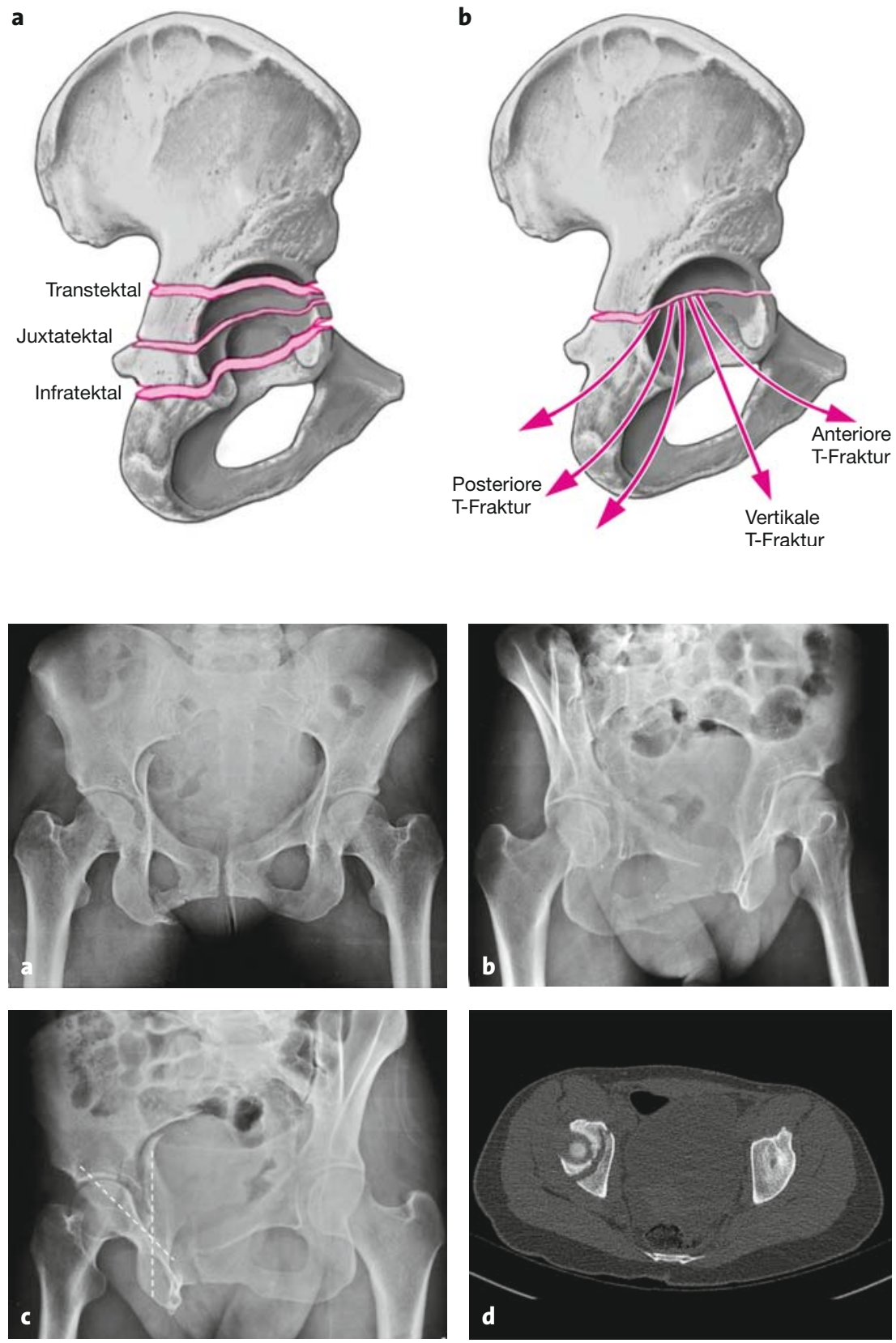

\section{Abbildungen 2a bis 2d}

a) Radiologische Präsentation einer Azetabulum-T-Fraktur bei einer 33-jährigen Frau. Die ilioischiale und die iliopektineale Linie sind unterbrochen.

b) Obturatoraufnahme: Der vertikale Stiel des T verläuft durch das Foramen obturatum.

c) Die Überkreuzung des vorderen und hinteren Pfeilers ist auf der Alaaufnahme ansatzweise zu erkennen.

d) Die transversale Komponente verläuft in der axialen Schichtung der Computertomographie in anteroposteriorer Richtung. sowie der Kontur des Foramen obturatum, außer dann, wenn der Stiel des $\mathrm{T}$ posterior durch das Tuber ischiadicum verläuft. Falls die vertikale Frakturlinie konventionell radiologisch nicht sicher identifiziert werden kann, sind folgende indirekte Zeichen hilfreich: 1. Überlappung der inferioren Fragmente beider Säulen im anteroposterioren Strahlengang, 2. Dislokation der Tränenfigur relativ zur ilioischialen Linie und 3. eine X-förmige Überlappung beider Säulen auf der Alaaufnahme (Abbildung 2c). Im Computertomo- gramm verläuft die Frakturlinie der transversalen Komponente in den axialen Schichten typischerweise in anteroposteriorer Richtung (Abbildung 2d).

Differentialdiagnostisch muss die posteriore T-Fraktur von einer transversalen Fraktur mit Beteiligung der Hinterwand unterschieden werden. Der Unterschied besteht darin, dass Frakturen der Hinterwand nicht bis in die quadrilaterale Fläche oder die Fossa acetabuli reichen und der inferiore Schambeinast intakt bleibt (Abbildungen $3 \mathrm{a}$ und $3 \mathrm{~b}$ ). 


\section{Abbildungen $3 a$ und $3 \mathbf{b}$}

Differentialdiagnose zwischen einer posterioren T-Fraktur und einer transversalen Azetabulumfraktur mit Beteiligung der Hinterwand (links dreidimensionales Schema; rechts zweidimensionale Präsentation analog einem axialen CT-Schnitt).

a) Relativ vertikal orientierte posteriore T-Fraktur.

b) Posteriore T-Fraktur.

(Position C:Transversale Azetabulumfraktur mit Beteiligung der Hinterwand.)

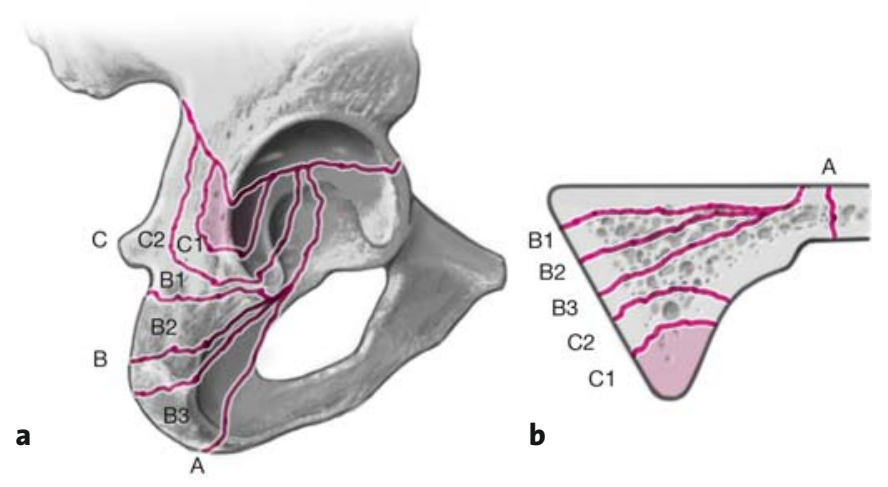

der beiden oder ein erweiterter iliofemoraler Zugang verwendet. Wir beschreiben zwei weichteilschonende Modifikationen der bisherigen Zugänge für die Osteosynthese von Azetabulum-T-Frakturen: die chirurgische Hüftluxation und den Stoppa-Zugang. Gegebenenfalls können beide Zugänge auch kombiniert werden.

Die chirurgische Hüftluxation als Zugang der Wahl wurde 2001 von Ganz et al. [2] beschrieben und erstmals von Siebenrock et al. für die Behandlung von Azetabulumfrakturen in einer Publikation zusammengefasst [7]. Diese Technik erlaubt die gefahrlose Luxation des Hüftkopfes mit einer vollständigen intraartikulären Visualisierung sowie einen zusätzlichen Zugang zum vorderen Azetabulum, was mit dem traditionellen KocherLangenbeck-Zugang nicht möglich ist. Für einige spezielle Indikationen kann auch der Stoppa-Zugang als intrapelviner Zugang verwendet werden, welcher zum ersten Mal 1994 von Cole \& Bolhofner beschrieben wurde [1].

\section{Operationsprinzip und -ziel}

Anatomische Reposition der Gelenkfragmente der Hüftpfanne und Stabilisierung mit (Zug-)Schrauben und Platten über einen posterioren Zugang mit chirurgischer Hüftluxation und/oder über einen anterioren Zugang (Stoppa-Zugang). Die Wahl des Zugangs richtet sich nach dem Ausmaß der Dislokation der Pfeiler: Chirurgische Hüftluxation bei stark disloziertem hinterem Pfeiler, Verwendung des Stoppa-Zugangs bei stark disloziertem vorderem Pfeiler (Abbildung 4). 


\section{Abbildung 4}

Die Wahl des Zugangs richtet sich nach dem Ausmaß der Dislokation. Eine überwiegende Dislokation des vorderen Pfeilers (Pfeil) mit einer Frakturöffnung nach ventral erfordert meist einen ventralen Stoppa-Zugang. Für eine vorwiegende Dislokation des hinteren Pfeilers mit einer Frakturöffnung nach dorsal (Pfeile) wird ein Zugang via chirurgische Hüftluxation empfohlen.

\section{Vorteile}

\section{Chirurgische Hüftluxation}

- Schonender intermuskulärer Zugang.

- Direkte intraartikuläre Visualisierung der gesamten azetabulären und femoralen Gelenkfläche.

- Zugang zur gesamten dorsalen Säule und zum kranialen Anteil des Azetabulums.

- Zusätzlich Zugang zur Vorderwand und zum vorderen Pfeiler.

- Möglichkeit der Refixation des Labrums.

- Möglichkeit der Therapie von Verletzungen des Femurkopfes.

- Möglichkeit der anatomischen Reposition bei zusätzlich impaktiertem Domfragment.

- Möglichkeit der Implantation einer primären Hüftprothese über denselben Zugang.

- Direkter visueller Ausschluss einer intraartikulären Schraubenlage.

\section{Stoppa-Zugang}

- Schnelle Rehabilitation.

- Relativ einfache und sichere chirurgische Präparation des Zugangs.

- Weniger invasiv als der klassische ilioinguinale Zugang oder der ausgedehnte iliofemorale Zugang.

- Einfacher Wundverschluss.

- Sehr geringe Tendenz zu heterotopen Ossifikationen.

- Keine Verletzungsgefahr des Nervus ischiadicus sowie des Nervus cutaneus femoris lateralis.

- Geringes Risiko einer Verletzung der großen iliakalen Gefäße.

- Kann zu einem vollständigen ilioinguinalen Zugang erweitert werden.

- Geringerer Blutverlust.

\section{Nachteile}

\section{Chirurgische Hüftluxation}

- Nur beschränkte Reposition des vorderen Pfeilers möglich.
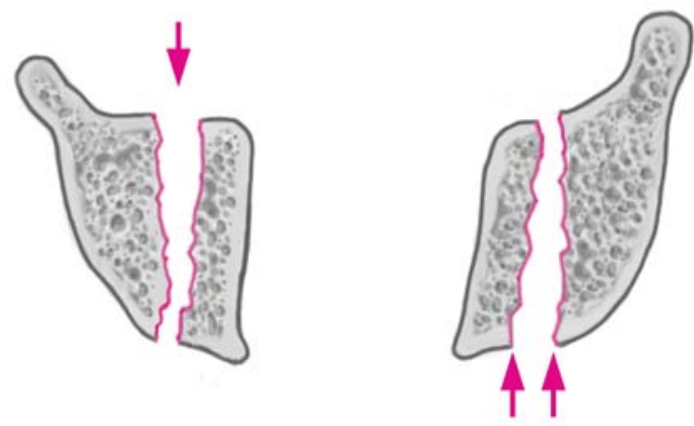

- Trochanterosteotomie notwendig.

- Minimale Gefahr einer avaskulären Nekrose des Hüftkopfes.

\section{Stoppa-Zugang}

- Teilweise schwierige Osteosynthese.

- Nur limitierte Reposition des hinteren Pfeilers möglich.

- Keine Objektivierung des intraartikulären Schadens.

- Keine Möglichkeit der gleichzeitigen primären Prothesenimplantation über denselben Zugang.

\section{Indikationen}

Chirurgische Hüftluxation

- Größere Dislokation des hinteren im Vergleich zum vorderen Pfeiler mit nach posterior offenem transversalem Frakturspalt (s. Abbildung 4).

- Minimale Dislokation des vorderen Pfeilers; die Stabilisierung des vorderen Pfeilers mit Zugschrauben ist möglich.

- Je kranialer die transversale Komponente der T-Fraktur, desto eher sollte ein posteriorer Zugang mit chirurgischer Luxation des Hüftgelenks zur anatomischen Reposition in Erwägung gezogen werden.

\section{Stoppa-Zugang}

- Minimale Dislokation des hinteren Pfeilers.

- Größere Dislokation des vorderen im Vergleich zum hinteren Pfeiler mit nach anterior offenem transversalem Frakturspalt (s. Abbildung 4).

\section{Kontraindikationen}

Chirurgische Hüftluxation

- Weit dislozierter vorderer Pfeiler.

\section{Stoppa-Zugang}

- Abdominale Problematik, inbesondere Ileus oder begleitende Verletzungen.

- Alter der Fraktur > 15 Tage. 
- Transtektale Frakturlinie: Hier sollte eine chirurgische Hüftluxation als Zugang gewählt werden, um eine direkte Visualisierung der Reposition der Hauptbelastungszone zu gewährleisten.

- Suprapubischer Blasenkatheter.

\section{Patientenaufklärung} Allgemeine Risiken

- Thrombose, Embolie, Wundinfektion, Hämatom.

- Bei relevantem Blutverlust Gabe von Blutkonserven.

- Entwicklung einer posttraumatischen Koxarthrose: T-Frakturen haben die schlechteste Prognose aller Azetabulumfrakturen.

- Mögliche Notwendigkeit von zwei chirurgischen Zugängen. Frakturen, die über zwei Zugänge angegangen werden müssen, sollten einzeitig operiert werden, da u.U. Osteosynthesematerial der zuerst rekonstruierten Säule eine anatomische Reposition der zweiten Säule behindern kann.

- Mögliche Notwendigkeit einer primären Hüftprothese, je nach Ausmaß des sichtbaren intraartikulären Schadens.

\section{Chirurgische Hüftluxation}

- Neurapraxie des Nervus ischiadicus.

- Trochanterpseudarthrose.

- Heterotope Ossifikationen.

- Reithosendeformität des subkutanen Gewebes.

- Minimales Risiko einer iatrogenen Hüftkopfnekrose.

\section{Stoppa-Zugang}

- Verletzung der Peritoneums oder der Harnblase.

- Neurapraxie des Nervus obturatorius.

- Entwicklung einer Inguinalhernie.

\section{Nachbehandlung}

- Rasche postoperative Mobilisation in der passiven Bewegungsschiene zur Verhinderung von intraartikulären Adhäsionen.

- Hospitalisationsdauer ca. 5-10 Tage (bei Monotraumen).

- 8 Wochen Teilbelastung von 5-10 kg der betroffenen Extremität an zwei Unterarmgehstöcken mit Thromboseprophylaxe.

- Maximale Flexion 90.

- Nach chirurgischer Hüftluxation keine aktive Abduktion, keine passive Adduktion, kein Überkreuzen der Beine während den ersten 6-8 postoperativen Wochen.
- Wiederaufnahme der Arbeitstätigkeit je nach Aktivitätsgrad nach 2-4 Monaten.

- Das definitive Ergebnis hängt direkt mit dem Ausmaß des traumatischen intraartikulären Schadens und der Genauigkeit der anatomischen Rekonstruktion zusammen.

\section{Operationsvorbereitungen}

- Standardröntgenbilder: Anteroposteriore Beckenübersicht, Ala- und Obturatoraufnahme. Die Abbildung der gesunden Gegenseite kann zu Vergleichszwecken sehr hilfreich sein.

- Präoperatives Computertomogramm mit dreidimensionalen Rekonstruktionen.

- Sorgfältige präoperative Planung des Zugangs, der Repositionstechniken und der anschließenden Osteosynthese.

- Blasendauerkatheter.

- Rasur des Operationsgebiets.

- Perioperative Antibiotikaprophylaxe mit Cefuroxim $(3 \times 1 \mathrm{~g}$ i.v./Tag), insgesamt 2 Tage postoperativ.

\section{Instrumentarium und Implantate}

- Knochen-, Weichteil- und Bauchsieb.

- Oszillierende Säge.

- Eventuell röntgendurchlässiger Operationstisch.

- Bildverstärker oder intraoperatives Röntgen.

- Beckengrundinstrumentarium, Beckenrepositionsinstrumentarium, kolineare Repositionszange (Synthes GmbH, Eimattstraße 3, 4436 Oberdorf, Schweiz).

- Zusätzlich oder alternativ: Matta pelvic system (Stryker Osteonics SA, C.P. 916, 5 Ch. des Aulx, 1212 Grand-Lancy I, Schweiz).

- In den meisten Fällen genügen Kleinfragmentimplantate mit selbstschneidenden 3,5-mm-Kortikalisschrauben zur Fixation. 4,5-mm-Kortikalisschrauben und Kleinfragmentimplantate werden nur selten benötigt.

\section{Anästhesie und Lagerung}

\section{Chirurgische Hüftluxation}

- Allgemeinanästhie mit guter intraoperativer Relaxation.

- Seitenlage.

\section{Stoppa-Zugang}

- Allgemeinanästhie mit guter intraoperativer Relaxation.

- Rückenlage. 


\title{
Operationstechnik
}

\author{
Abbildungen 5 bis 27
}

\section{Offene Reposition und Osteosynthese via chirurgische Hüftluxation (Abbildungen 5 bis 18)}

\section{Abbildung 5}

Das gesamte Bein wird beweglich steril abgedeckt. Der Operateur steht dorsal des Patienten. Die Hautinzision verläuft lateral gerade zentriert über dem Trochanter major auf einer Länge von ca. $20 \mathrm{~cm}$.

\begin{abstract}
Abbildung 6
Proximal wird das Gibson-Intervall zwischen den Musculi glutei medius und maximus aufgesucht und gespalten. Als anatomische Landmarke dienen hierzu die Vasa perforantia, welche zwischen diesen beiden Muskeln verlaufen. Die Vasa perforantia werden derart präpariert, dass sie in einer Faszienschicht auf der Innenseite des Musculus gluteus maximus verlaufen. Distal wird die Fascia lata längs inzidiert, und nach Inzision der Bursa trochanterica wird der posteriore Anteil des Musculus gluteus medius und des Musculus vastus lateralis bei innenrotiertem Bein aufgesucht. Der Musculus vastus lateralis wird an seinem dorsalen Rand inzidiert und angehoben.
\end{abstract}
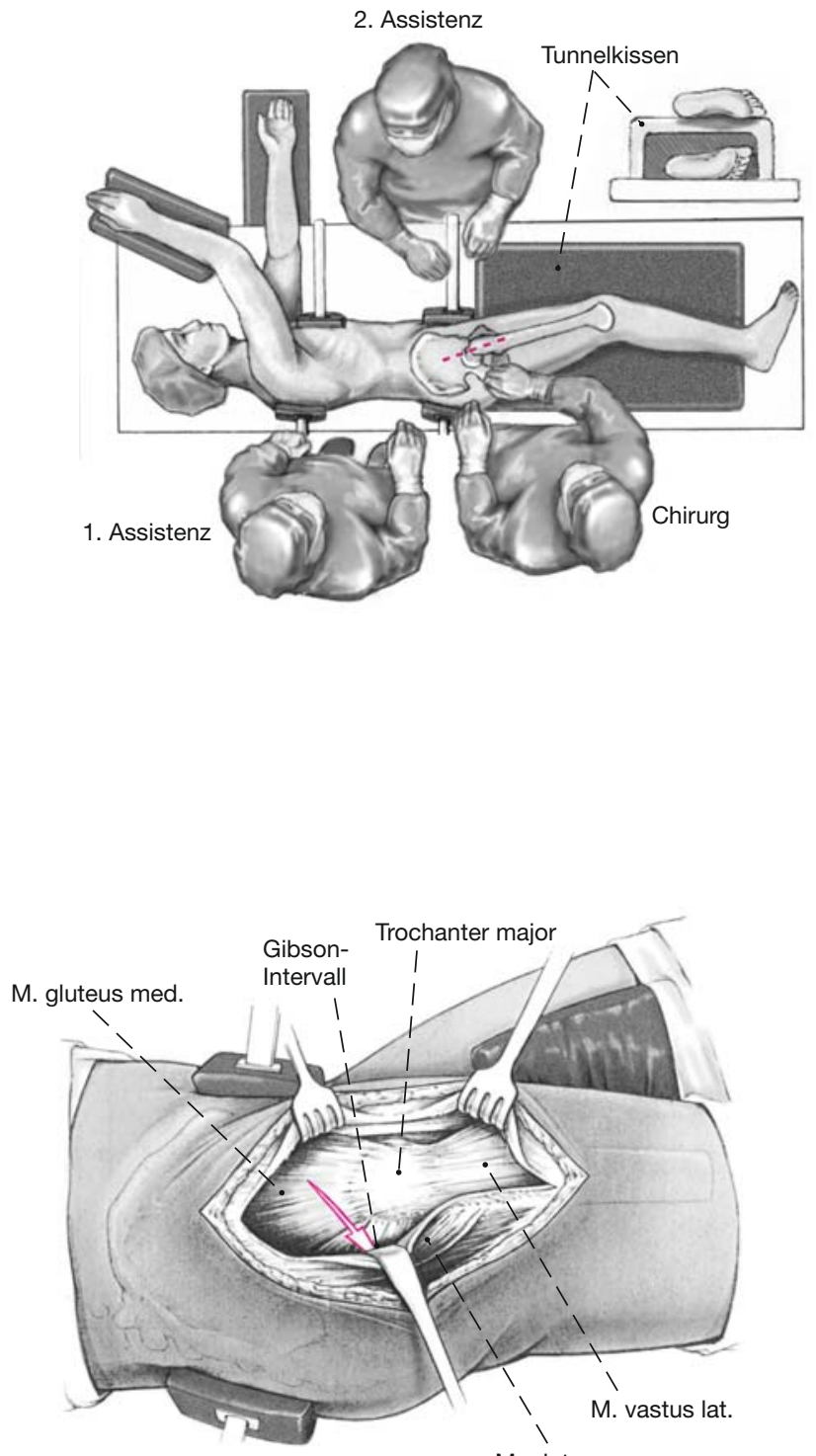

M. gluteus max 


\section{Abbildung 7}

Anschließend zeigt sich ein konstanter Trochanterast der Arteria circumflexa femoris medialis, welcher kranial des Musculus quadratus femoris und kaudal des Musculus triceps coxae verläuft. Danach erfolgt eine gerade oder Z-förmige Osteotomie des Trochanter major mit der oszillierenden Säge. Um eine zu mediale Osteotomie und dadurch eine Verletzung der Arteria circumflexa femoris medialis (arterielle Hauptversorgung des Femurkopfes) zu vermeiden, wird ein kleiner Streifen des dorsalen Sehnenanteils des Musculus gluteus medius zunächst am proximalen Femur belassen (Pfeil). Der distale Zielpunkt der Osteotomie liegt mediokaudal des Tuberculum innominatum. Der letzte Anteil der Kortikalis sollte anschließend mit geraden Meißeln kontrolliert frakturiert werden. Dies schafft ideale Referenzen für eine spätere anatomische Refixation. Nach der Osteotomie können die belassenen Fasern des Musculus gluteus medius scharf durchtrennt werden, so dass sich das Trochanterfragment nach ventral verschieben lässt.

\section{Abbildung 8}

Der tiefe Ast der Arteria circumflexa femoris medialis ist durch die kleinen Außenrotatoren geschützt. Das Trochantermedaillon wird weiter nach ventral mobilisiert, indem die Musculi gluteus minimus und vastus lateralis von der darunterliegenden Kapsel und dem Knochen abgelöst werden. Von dorsal erscheint zuerst der Musculus piriformis und anschließend der Musculus gluteus minimus.
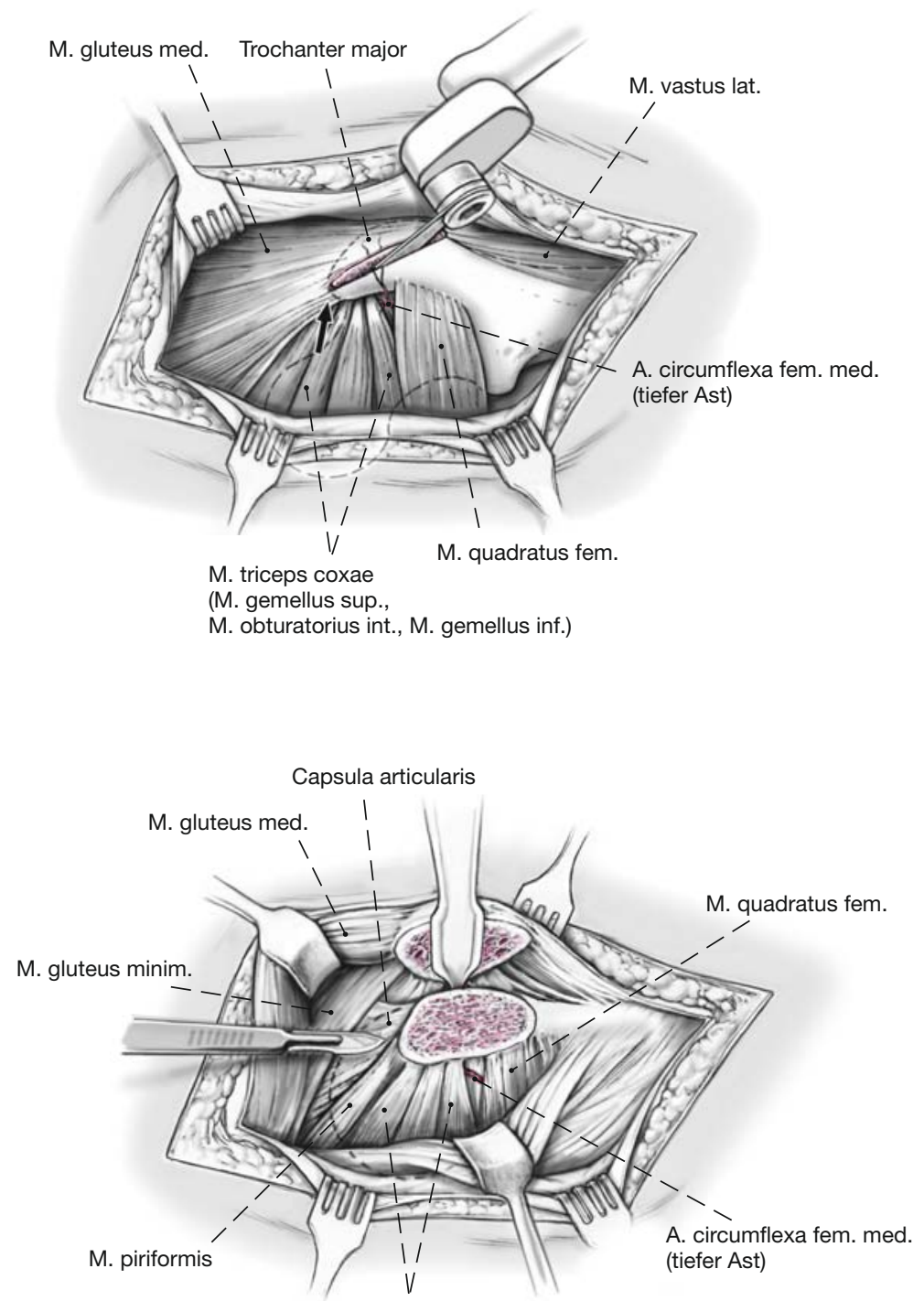

M. triceps coxae

\section{Abbildung 9}

Durch das Intervall zwischen dem Musculus gluteus minimus und dem Musculus piriformis wird die Gelenkkapsel präpariert.

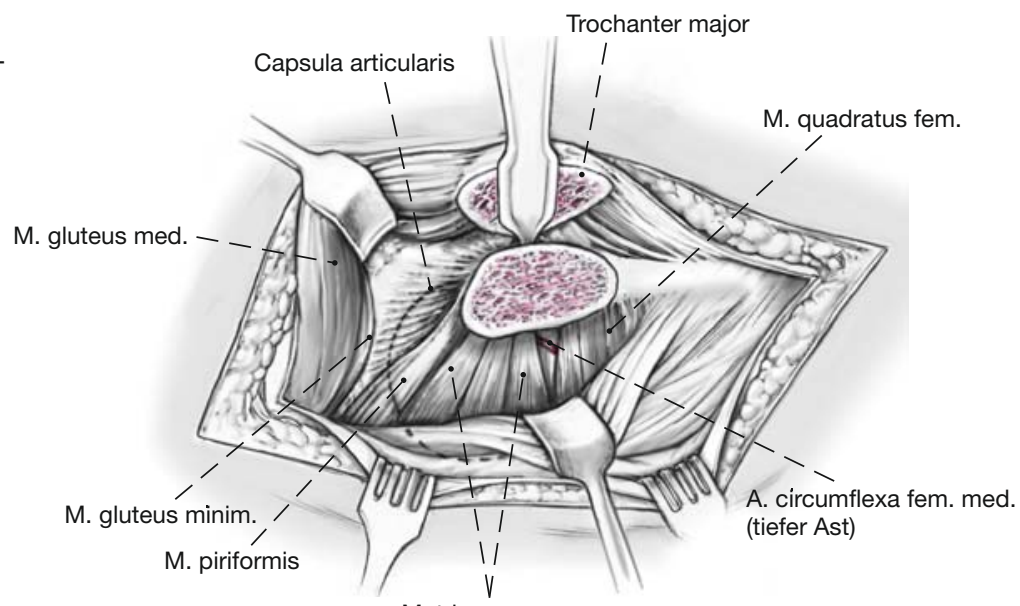

M. triceps coxae 


\section{Abbildung 10}

Die Präparation der ventralen Gelenkkapsel wird durch Flexion und Außenrotation des Beins erleichtert.

\section{Abbildung 11}

Die Gelenkkapsel wird Z-förmig eröffnet.

\section{Abbildung 12}

Durch Außenrotation, Adduktion und Flexion kann nach Durchtrennung des Ligamentum capitis femoris mit einer gebogenen Schere der Femurkopf mit Hilfe eines Einzinkerhakens luxiert werden.
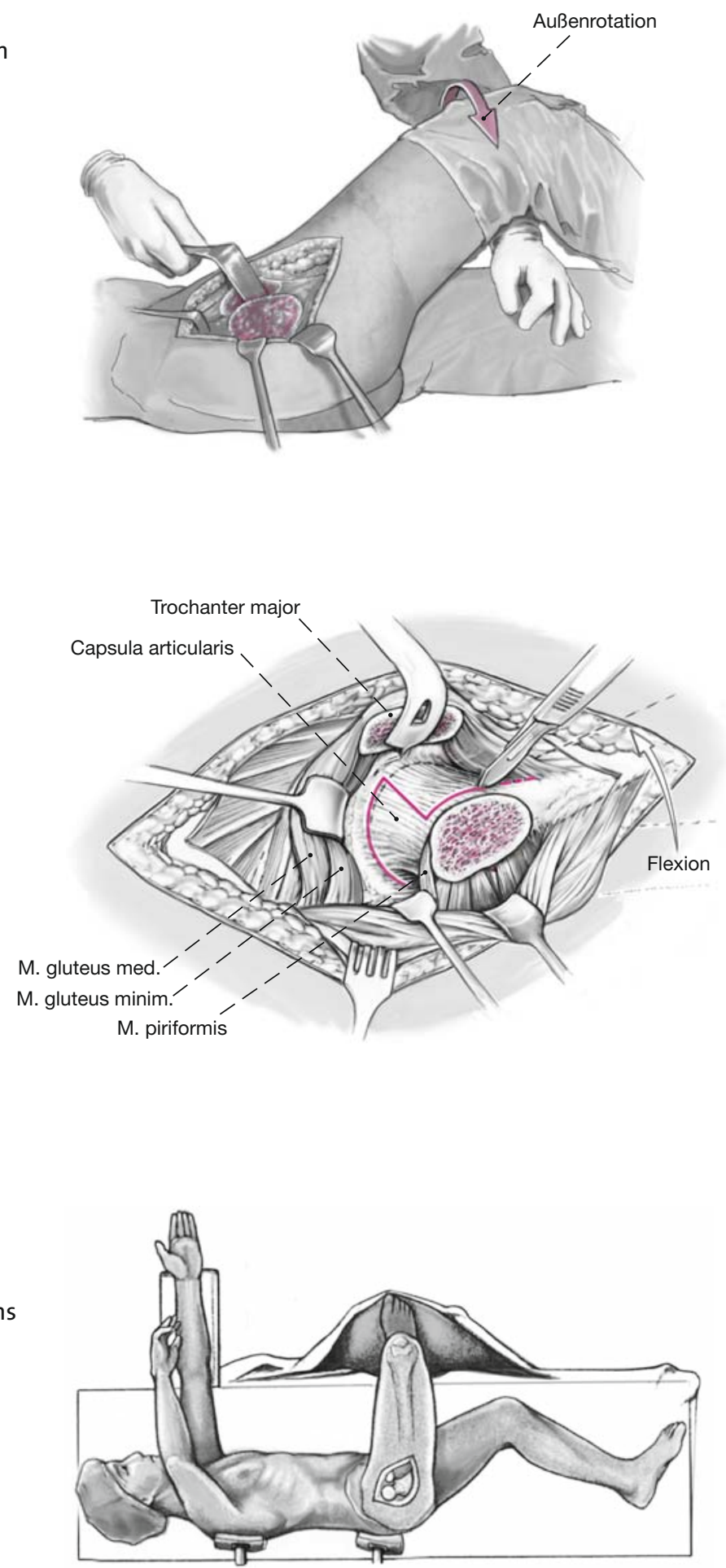


\section{Abbildung 13}

Zur Darstellung des Gelenks wird meist ein Retraktor über den vorderen Pfeiler eingesetzt und durch einen weiteren Retraktor in der Incisura acetabuli das Calcar femorale retrahiert. Der Zugang erlaubt eine vollständige Visualisierung der intraartikulären Frakturkonfiguration sowie eine Quantifizierung des Schadensmusters an Knorpel und Labrum.

\section{Abbildung 14}

Intraoperativer Situs bei luxierter Hüfte (gleiche Patientin wie in Abbildung 2). Blick auf die transtektale, vertikale T-Fraktur.
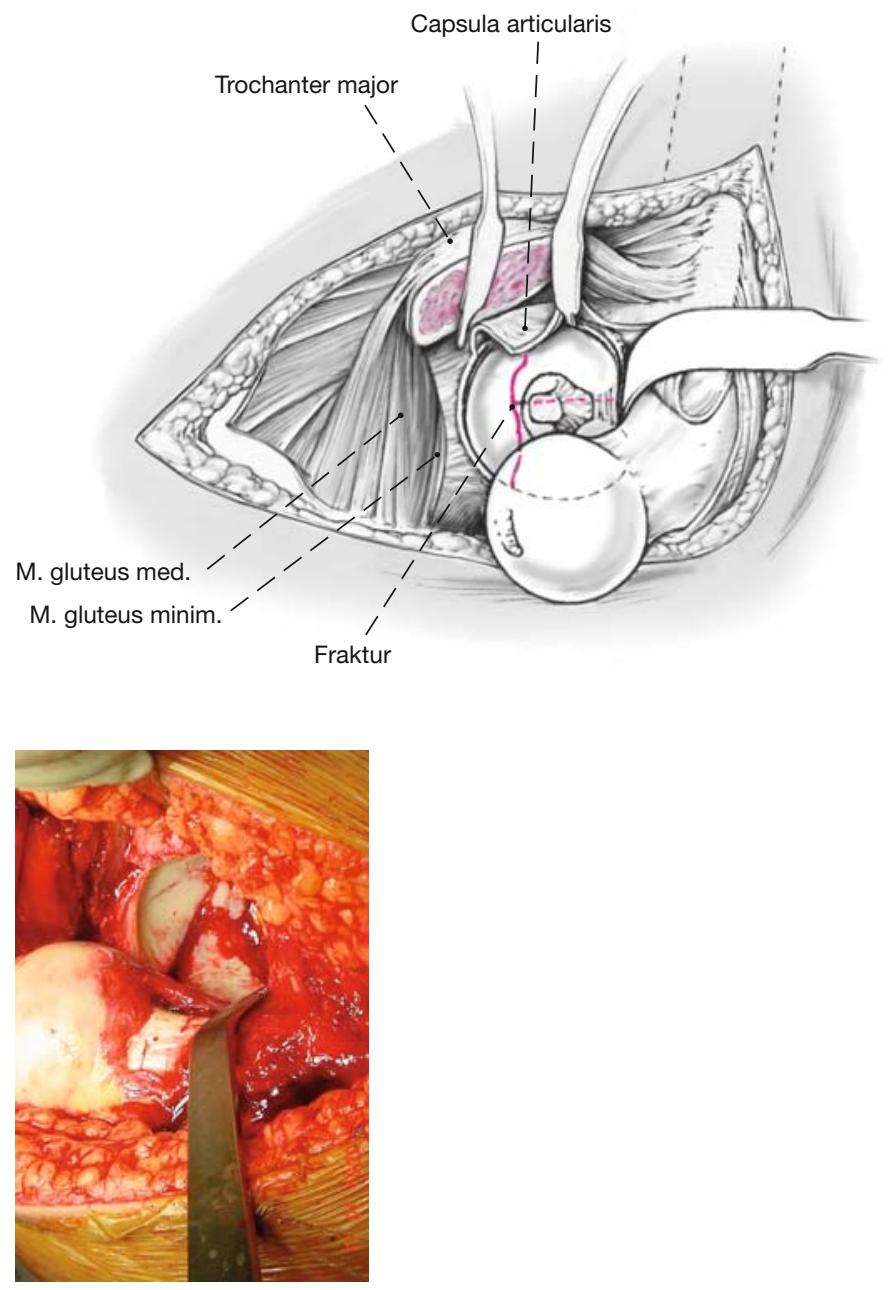

a

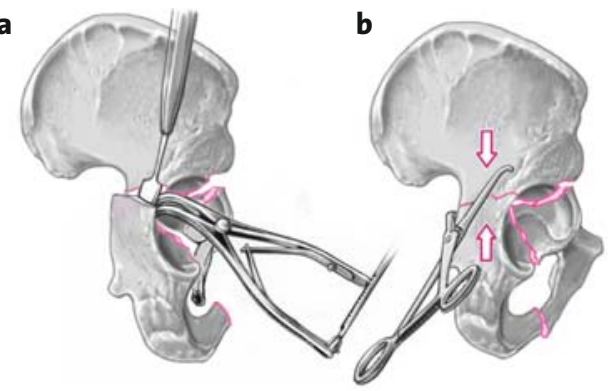

d

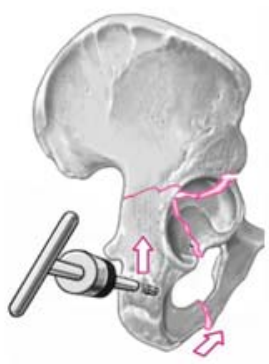

e

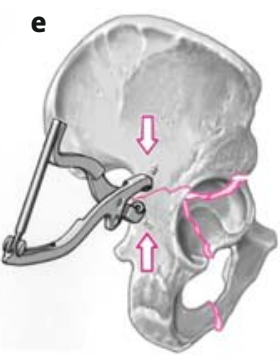

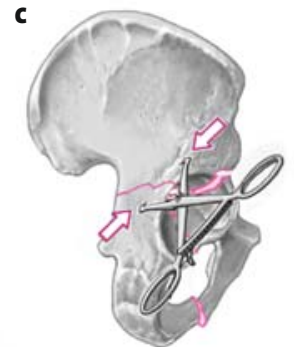

C

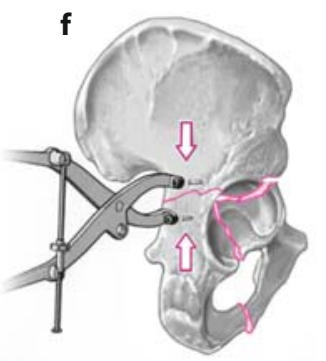




\section{Abbildung 16}

Der hintere Pfeiler kann mit Zugschrauben, anschließender Neutralisierungsplatte oder direkt über eine angebogene Rekonstruktionsplatte fixiert werden. Der vordere Pfeiler wird entweder direkt mit einem Einzinkerhaken oder durch Manipulationen mit einem Hohmann-Haken reponiert. Die Haken werden dabei über die vordere azetabuläre Lippe angesetzt. Mit einer nicht ganz vorkonturierten Platte kann zudem zusätzliche Kompression der Fragmente erreicht werden.
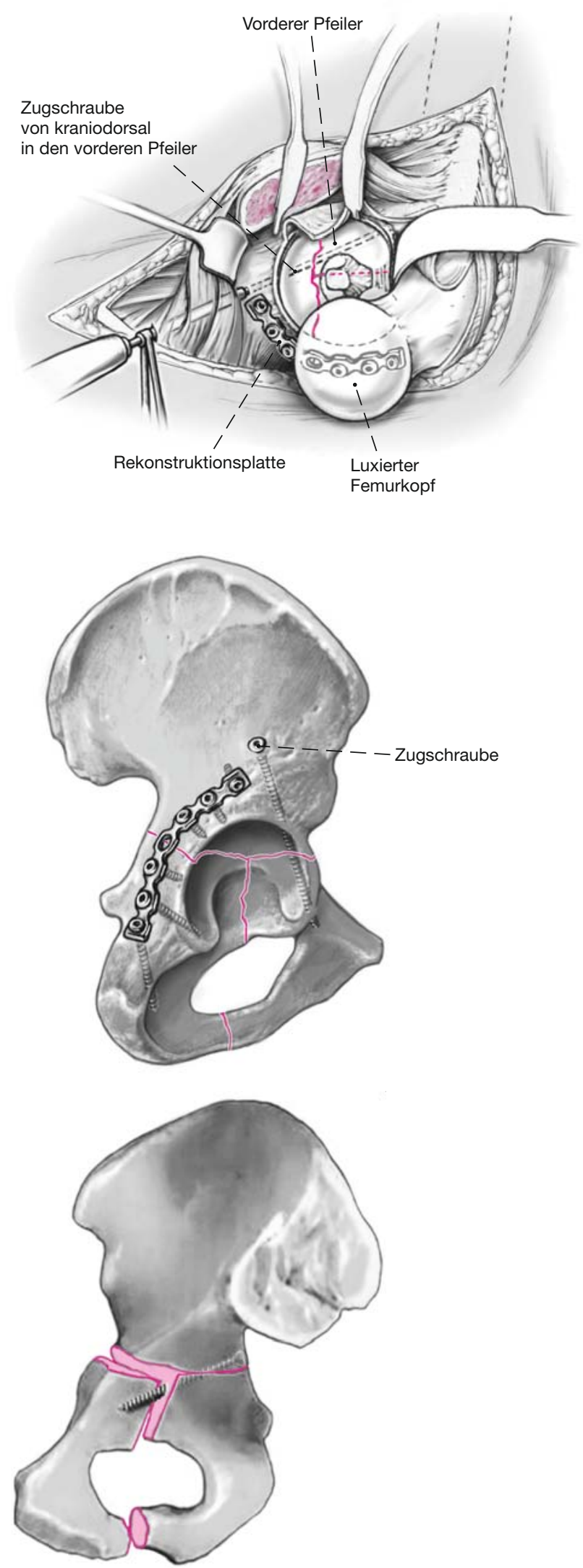

\section{Abbildung 17}

Eine von kraniodorsal in den vorderen Pfeiler in Richtung des Schambeinasts eingebrachte Zug- oder Stellschraube fixiert diesen Teil der Fraktur. Eine intraartikuläre Perforation kann durch optische Kontrolle ausgeschlossen werden.

\section{Abbildung 18}

Es ist darauf zu achten, dass die Schrauben zur Fixation des hinteren Pfeilers die Reposition des vorderen Pfeilers nicht behindern. Nach Reposition und Stabilisierung schrittweiser Wundverschluss. Die Gelenkkapsel wird mit einem resorbierbaren Faden genäht. Anschließend wird das TrochantermedailIon mit zwei 3,5-mm-Kortikalisschrauben refixiert, und der Tractus iliotibialis wird mit einer resorbierbaren PDS-Kordel verschlossen. Subkutan- und Kutannaht. Einlage einer tiefen und einer oberflächlichen Drainage. Steriler Verband mit Longuetten und einem Fixiervlies. 


\section{Offene Reposition und Osteosynthese via Stoppa-Zugang (Abbildungen 19 bis 27)}

\section{Abbildung 19}

Das gesamte Hypogastrium inklusive der Spinae iliacae anteriores superiores und der Symphyse wird abgedeckt. Das ipsilaterale Bein wird beweglich abgedeckt. Der Operateur steht auf der der Fraktur gegenüberliegenden Seite. Transversale, gerade oder leicht geschwungene Inzision ca. $2 \mathrm{~cm}$ kranial der Symphyse über eine Länge von etwa $15 \mathrm{~cm}$.

\section{Abbildung 20}

Nach Präparation des subkutanen Fettgewebes wird die vordere Rektusscheide identifiziert und längs gespalten.

\section{Abbildung 21}

Der Musculus rectus abdominis und der Musculus pyramidalis werden identifiziert, und das Intervall zwischen den beiden Muskelbäuchen wird auf einer Länge von ca. $15 \mathrm{~cm}$ längs gespalten, wodurch das extraperitoneale Fett zum Vorschein kommt. Es ist sorgfältig darauf zu achten, das Peritoneum vor allem im kranialen Teil des Situs nicht zu verletzen.
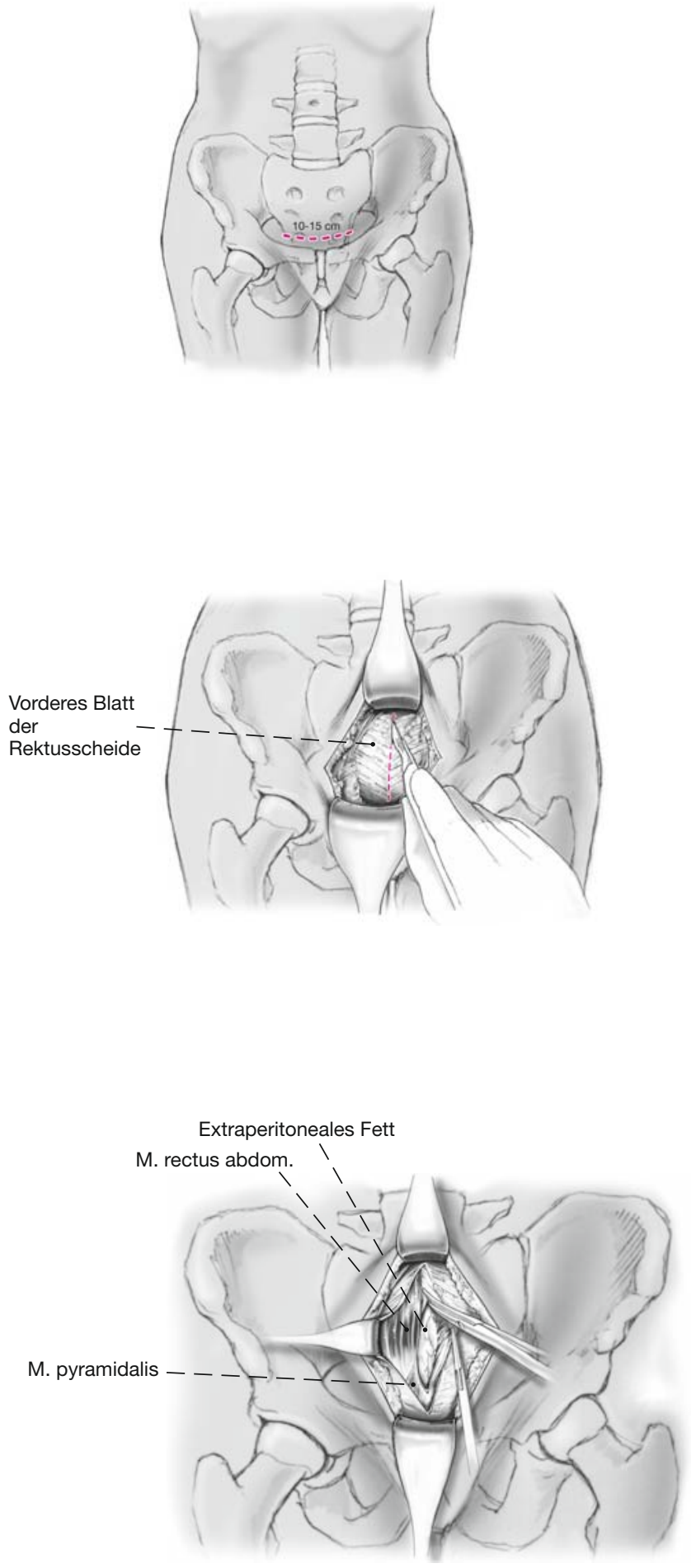


\section{Abbildung 22}

Durch stumpfe Präparation des extraperitonealen Fetts mit einem Stieltupfer wird der prävesikale Retzius-Raum präpariert, und die Harnblase wird mit einem Bauchspatel geschützt. Bei liegendem Dauerkatheter kann bei klarem Urin eine Verletzung der Harnblase ausgeschlossen werden. Bei einer allfälligen Verletzung der Harnblase kann diese über denselben Zugang direkt angegangen werden.

\section{Abbildung 23}

Der Ansatz des Musculus rectus abdominis wird vom er ipsilateral auch komplett scharf von seinem Ansatz abgetrennt werden. Weitere stumpfe Präparation nach lateral entlang dem Pecten ossis pubis unter Ablösung des kraniomedialen Anteils des Musculus pectineus bis zur Linea arcuata. Meist zeigt sich eine kräftige arteriovenöse Anastomose zwischen der Arteria obturatoria und der Arteria epigastrica inferior an der Innenfläche des Schambeinasts [3, 5, 6]. Diese als „Corona mortis“ bekannte Konfiguration sollte freipräpariert, unterbunden und durchtrennt werden, um eine lebensgefährliche Blutung zu vermeiden. oberen Schambeinast mobilisiert. Falls notwendig, kann
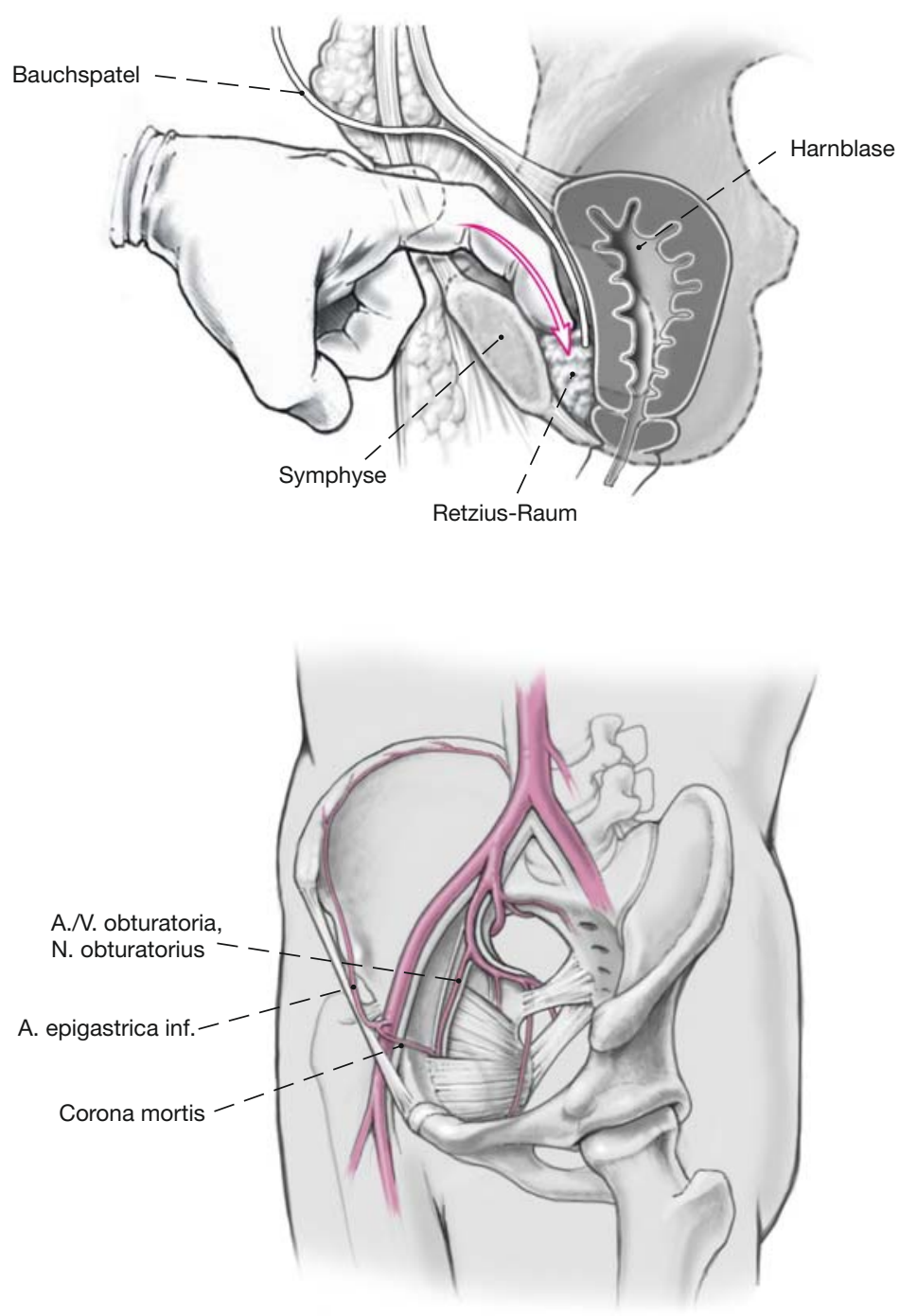

\section{Abbildung 24}

Die Vasa obturatoria und der Nervus obturatorius, welche durch beide Anteile des Musculus obturatorius in den Canalis obturatorius einmünden, werden aufgesucht und geschont. Die weitere Präparation und Darstellung der Fraktur erfolgen viszeral der iliakalen Gefäße, des Nervus femoralis und des Musculus iliopsoas, welche mit einem Haken über dem kranialen Schambeinast nach lateral retrahiert werden. Leichte Hüftflexion von ca. 30-45 auf einer Kniestütze entspannt den Musculus iliopsoas und erleichtert die weitere Präparation.

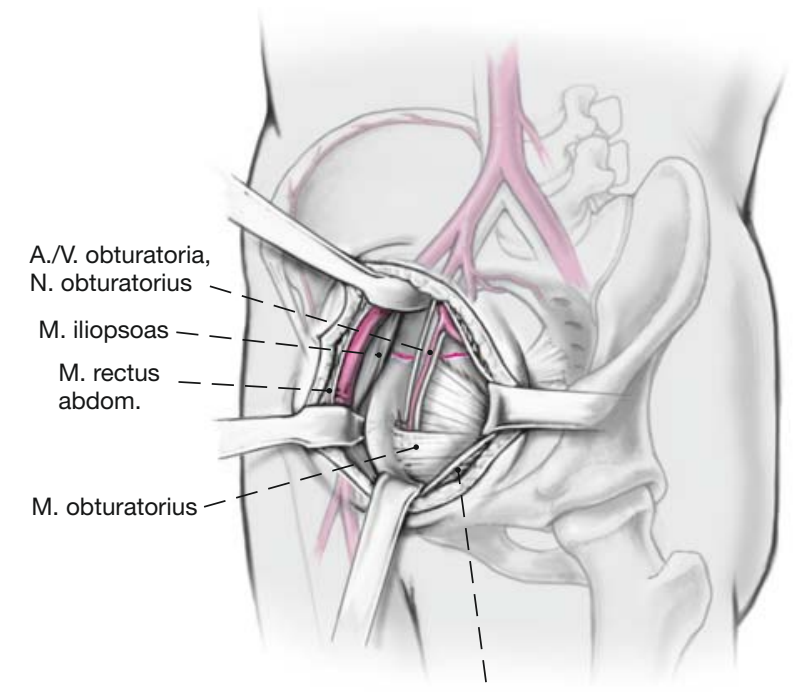

M. rectus abdom. 


\section{Abbildung 25}

Eine weitere stumpfe Elevation des Musculus iliopsoas eröffnet die Sicht auf die Fossa iliaca. Bevor der posteriore Anteil des Musculus iliacus mobilisiert wird, sollte der das Azetabulum versorgende Ramus iliacus der Arteria iliolumbalis ligiert oder koaguliert werden, falls er nicht bereits durch die Frakturränder durchtrennt ist. Dies verringert den Blutverlust aus der Fraktur und verbessert die Übersicht.

\begin{abstract}
Abbildung 26
Der Musculus obturatorius internus wird stumpf vom seinem Ursprung an der quadrilateralen Fläche abgelöst, was die transversale Komponente der T-Fraktur freilegt. Der Musculus obturatorius wird zusammen mit den Vasa obturatoria und dem Nervus obturatorius mit einem Bauchspatel sorgfältig nach medial retrahiert. Insgesamt erlaubt dieser Zugang eine vollständige Darstellung des oberen Schambeinasts, der quadrilateralen Fläche sowie der Linea arcuata bis hin zum Iliosakralgelenk. Sorgfältiges Freipräparieren der Frakturränder mit Ablösen des Periosts zur besseren Visualisierung und Auskürettieren der Frakturflächen. Je nach Frakturkonfiguration Reposition des vorderen Pfeilers in lateraler Richtung mit Stößeln oder einem Raspatorium, axialem Zug am Bein, Matta-Klemmen oder anderen Repositionszangen.
\end{abstract}
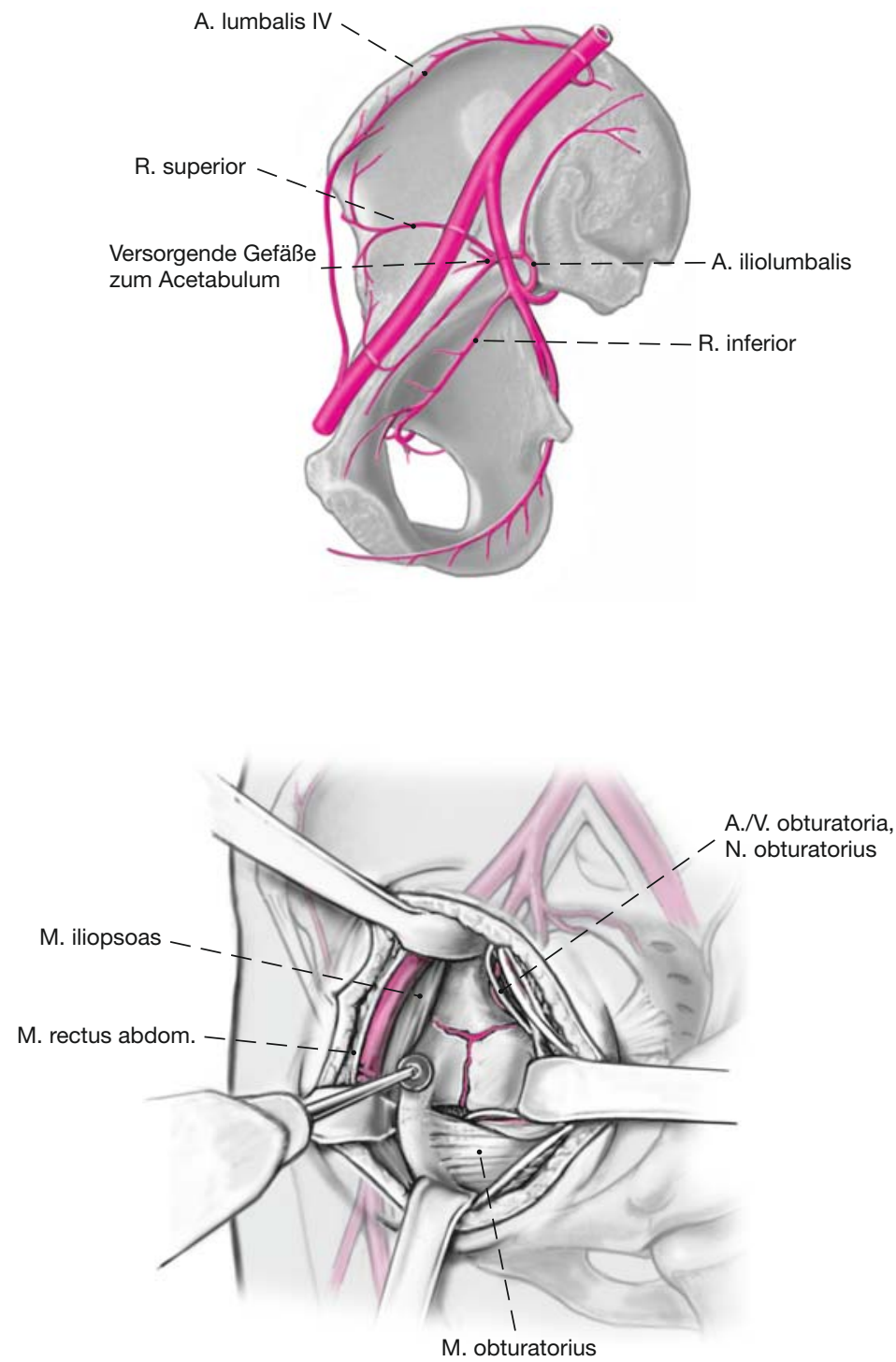


\section{Abbildungen 27a und $27 \mathrm{~b}$}

a) Der hintere Pfeiler kann mit einer kolinearen Zange durch das (später beschriebene) erste Fenster des ilioinguinalen Zugangs reponiert werden.

b) Die exakte Lage der Repositionsklemmen hängt dabei von der individuellen Frakturkonfiguration ab. Ziel ist es, eine anatomische Reposition und provisorische Fixation des vorderen Pfeilers z.B. mit Kirschner-Drähten zu erreichen und anschließend den hinteren Pfeiler mit Zugschrauben zu fassen. Die Neutralisationsplatte kann dabei je nach Frakturkonfiguration supra- oder infrapektineal angelegt werden. Falls sich keine anatomische Reposition des hinteren Pfeilers erreichen lässt, muss er über einen zusätzlichen dorsalen Zugang nach Umlagerung reponiert und stabilisiert werden.

Nach vollständiger Fixierung der Fraktur wird der allenfalls abgelöste Musculus rectus abdominis wieder transossär am Schambeinast refixiert. Die Rektusscheide wird mit einem resorbierbaren Faden verschlossen. Einlage einer Drainage in den Retzius-Raum. Subkutan- und Kutannaht. Steriler Verband mit Longuetten und einem Fixiervlies.

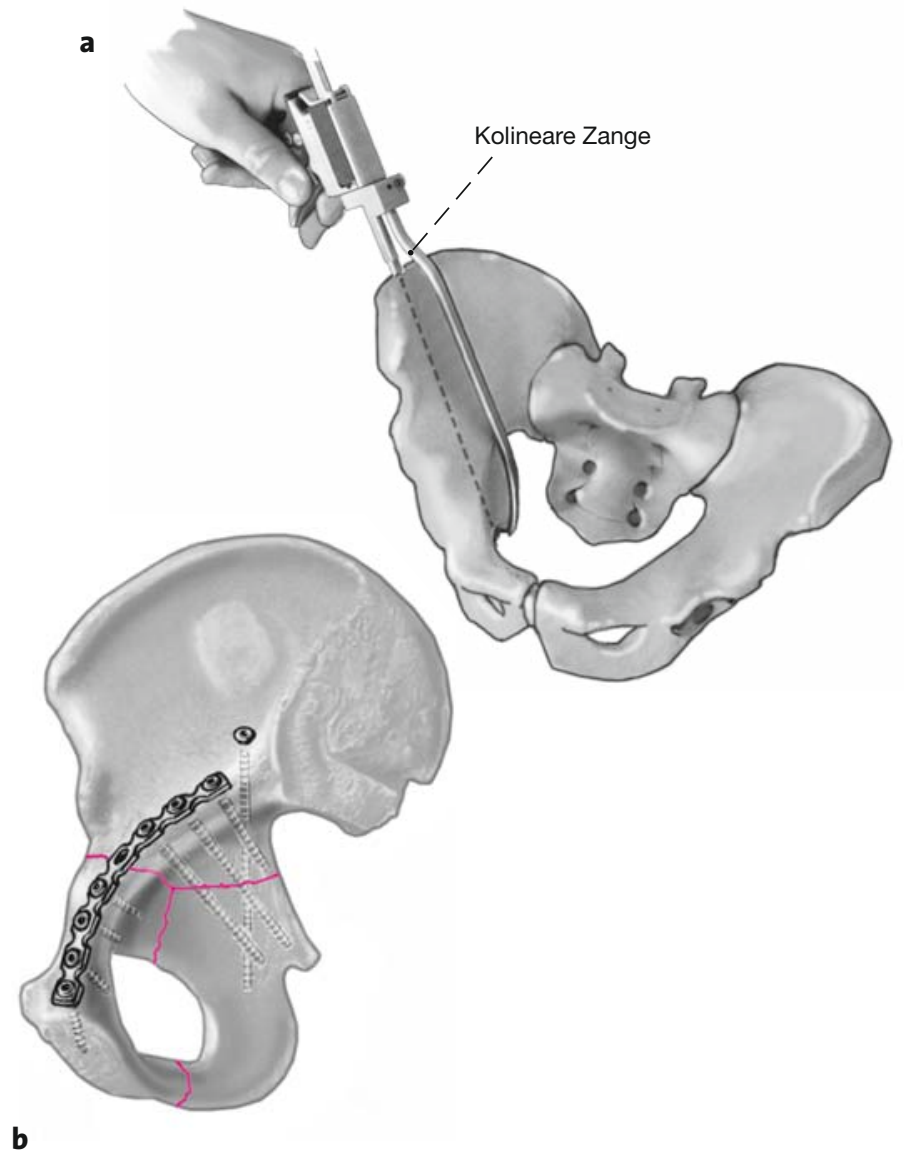

b

\section{Besonderheiten (Abbildungen 28 und 29)}

Bei ausgedehntem intraartikulärem Schaden, der nach Luxation des Femurkopfes bei einer chirurgischen Hüftluxation sichtbar wird, kann über diesen Zugang nach Reposition und Osteosynthese auch primär eine Hüftprothese eingesetzt werden.

\section{Abbildung $\mathbf{2 8}$}

Ist mit dem Stoppa-Zugang keine Schraubeneinbringung in gewünschter Richtung in den hinteren Pfeiler möglich, wird zusätzlich das erste Fenster des klassischen ilioinguinalen Zugangs eröffnet. Hierfür wird eine etwa $10 \mathrm{~cm}$ lange Inzision, ausgehend von der Spina iliaca anterior superior, entlang dem Beckenkamm nach dorsal angelegt.

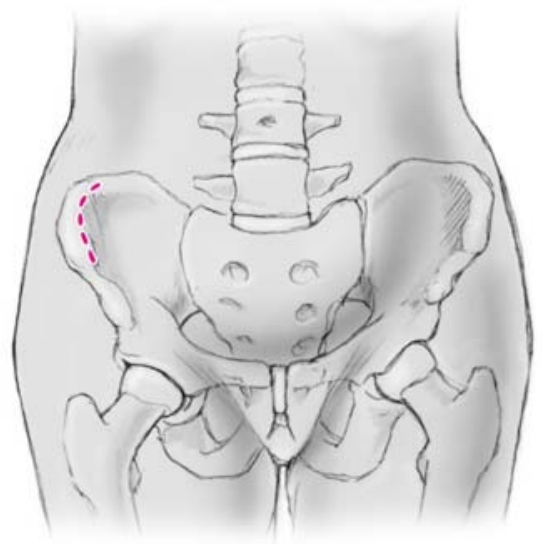




\section{Abbildung 29}

Nach Dissektion der Subkutis werden die gemeinsamen Insertionen der Abdominalmuskeln und der Hüftabduktoren entlang dem Beckenkamm inzidiert. Die Darstellung der Fossa iliaca interna wird durch subperiostale Ablösung des Musculus iliacus komplettiert, wodurch die eingeschobene Platte sichtbar werden sollte. Nach Reposition des hinteren Pfeilers Einbringen der ein bis zwei Zugschrauben von kraniolateral. Wundverschluss über transossäre Refixation der Bauchwandmuskulatur. Einlage einer Redon-Drainage. Subkutan- und Kutannaht.

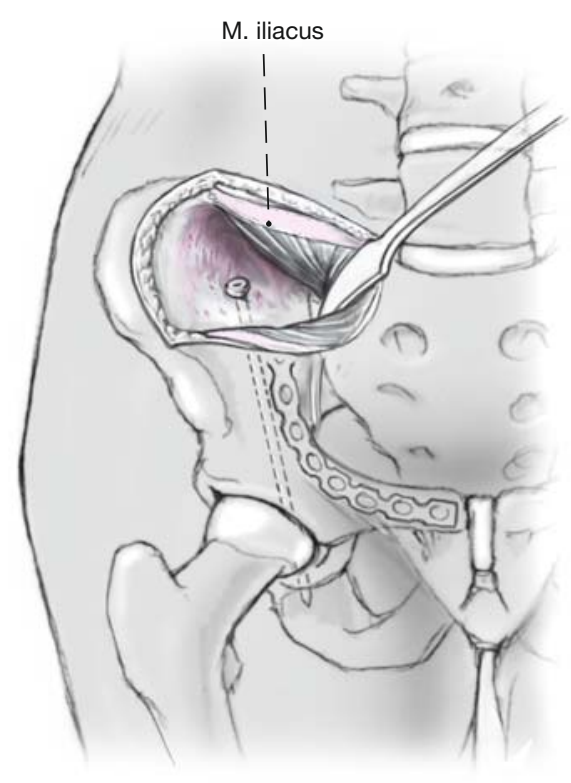

- Störende Trochanterschrauben sollten frühestens nach 6 Monaten entfernt werden; das Osteosynthesematerial periazetabulär wird in der Regel belassen.

\section{Fehler, Gefahren, Komplikationen}

- Peritoneumperforation: Intraoperative Naht des Peritoneums.

- Verletzung der Harnblase: Primäre Naht.

- Intraoperative Verletzung der hüftkopfernährenden Gefäße: Mehrmaliges Anbohren des Hüftkopfes oder intraoperative Laser-Doppler-Messung der Hüftkopfdurchblutung. Bei Restperfusion keine Maßnahmen notwendig; bei Ausbleiben einer pulsativen Blutung respektive eines pulsativen Signals Wechsel auf primäre Hüftprothese.

- Verletzung des Nervus obturatorius mit Adduktorenschwäche und Dys-/Anästhesien im Versorgungsgebiet: Bei einer iatrogenen, akzidentellen Neurotmesis ist eine Nervennaht indiziert. Eine Neurapraxie infolge eines Traktionsschadens durch den Bauchspatel ist meist passager.

- Postoperative Darmatonie: Gabe von Darmstimulanzien.

- Trochanterpseudarthrose nach 8 Wochen: Vorerst Abwarten einer Spontanheilung mit Fortführung der Teilbelastung für weitere 4 Wochen. Bei ausbleibender Heilung Trochanterrevision.

- Auftreten von heterotopen Ossifikationen bei der ersten Verlaufskontrolle: Abwarten der Ausreifung der Ossifikationen, welche nach einigen Monaten abgeschlossen ist. Entfernung der Ossifikationen 
Tabelle 1

Ergebnisse. CHL: chirurgische Hüftluxation; M: männlich; W: weiblich.

\begin{tabular}{|c|c|c|c|c|c|c|c|c|c|c|c|}
\hline Patient & $\begin{array}{l}\text { Ge- } \\
\text { schlecht }\end{array}$ & $\begin{array}{l}\text { Alter } \\
\text { (Jahre) }\end{array}$ & Unfall & $\begin{array}{l}\text { Mono-/ } \\
\text { Polytrauma }\end{array}$ & $\begin{array}{l}\text { Traumatische } \\
\text { Luxation }\end{array}$ & Zugang & $\begin{array}{l}\text { Primär- } \\
\text { prothese }\end{array}$ & $\begin{array}{l}\text { Operations- } \\
\text { zeit (h) }\end{array}$ & Reposition & $\begin{array}{l}\text { Verlaufs- } \\
\text { kontrolle } \\
\text { (Jahre) }\end{array}$ & $\begin{array}{l}\text { Komplikationen/ } \\
\text { Reoperationen }\end{array}$ \\
\hline A.S. & M & 18,8 & Verkehrsunfall & Monotrauma & $\mathrm{Ja}$ & $\mathrm{CHL}$ & Nein & 4,5 & Anatomisch & 2,3 & Keine \\
\hline B.M. & M & 59,8 & Verkehrsunfall & Polytrauma & $\mathrm{Ja}$ & $\mathrm{CHL}$ & $\mathrm{Ja}$ & 2,4 & - & 0,4 & Keine \\
\hline B.M. & W & 77,3 & Sturz & Polytrauma & Nein & $\mathrm{CHL}$ & $\mathrm{Ja}$ & 3,0 & - & 2,0 & Keine \\
\hline B.K. & W & 53,4 & Verkehrsunfall & Polytrauma & Nein & $\begin{array}{l}\text { CHL + Stoppa/ } \\
\text { ilioinguinal } \\
\text { (zweizeitig) }\end{array}$ & Nein & 4,5 & Anatomisch & 2,3 & $\begin{array}{l}\text { Hüftprothese } 2,3 \\
\text { Jahre postoperativ }\end{array}$ \\
\hline B.M. & M & 19,9 & Sturz & Polytrauma & Nein & $\begin{array}{l}\text { CHL + Stoppa } \\
\text { (zweizeitig) }\end{array}$ & Nein & 4,0 & Anatomisch & 1,0 & Keine \\
\hline C.M. & W & 54,6 & Verkehrsunfall & Polytrauma & Nein & CHL + Stoppa & Nein & 10,0 & Anatomisch & 2,3 & $\begin{array}{l}\text { Heterotope Ossifika- } \\
\text { tionen Grad II-III }\end{array}$ \\
\hline C.D. & W & 33,0 & Unbekannt & Polytrauma & Nein & $\mathrm{CHL}$ & Nein & 3,0 & Anatomisch & 6,2 & Keine \\
\hline G.M. & M & 58,5 & Skiunfall & Monotrauma & Nein & Stoppa & Nein & 3,0 & Anatomisch & 2,4 & Repositionsverlust \\
\hline H.Y. & W & 82,7 & $\begin{array}{l}\text { Niederenergie- } \\
\text { trauma }\end{array}$ & Monotrauma & Nein & $\mathrm{CHL}$ & $\mathrm{Ja}$ & 3,0 & - & Keine & Keine \\
\hline K.M. & W & 64,0 & Skiunfall & Monotrauma & Nein & $\mathrm{CHL}$ & $\mathrm{Ja}$ & 2,5 & - & 7,0 & Keine \\
\hline L.C. & M & 34,9 & Verkehrsunfall & Monotrauma & $\mathrm{Ja}$ & $\mathrm{CHL}$ & Nein & 4,0 & Anatomisch & Keine & Keine \\
\hline L.G. & W & 51,4 & Sturz & Monotrauma & Nein & Ilioinguinal & Nein & 3,5 & Anatomisch & 2,5 & $\begin{array}{l}\text { Hüftprothese } \\
8 \text { Monate post- } \\
\text { operativ }\end{array}$ \\
\hline M.R. & W & 44,8 & Verkehrsunfall & Polytrauma & Nein & $\begin{array}{l}\text { CHL + Smith- } \\
\text { Petersen }\end{array}$ & Nein & 8,0 & $\begin{array}{l}\text { Zufrieden- } \\
\text { stellend }\end{array}$ & Keine & Keine \\
\hline N.W. & M & 74,6 & Skiunfall & Monotrauma & Nein & $\begin{array}{l}\text { Stoppa + } \\
\text { ilioinguinal }\end{array}$ & $\mathrm{Ja}$ & 5,0 & - & Keine & Keine \\
\hline S.H. & M & 55,7 & Unbekannt & Monotrauma & Nein & $\mathrm{CHL}$ & Nein & 1,5 & Anatomisch & 7,4 & $\begin{array}{l}\text { Verzögerte } \\
\text { Trochanterheilung }\end{array}$ \\
\hline T.D. & M & 20,7 & Verkehrsunfall & Polytrauma & Nein & $\mathrm{CHL}$ & Nein & 7,0 & Anatomisch & 2,3 & Keine \\
\hline Z.M. & M & 24,8 & Verkehrsunfall & Polytrauma & Nein & $\mathrm{CHL}$ & Nein & 1,5 & $\begin{array}{l}\text { Zufrieden- } \\
\text { stellend }\end{array}$ & Keine & Keine \\
\hline
\end{tabular}

nur bei eingeschränkter Beweglichkeit des Gelenks.

- Reithosendeformität des subkutanen Gewebes: Falls vom Patienten gewünscht, plastische Korrektur.

- Entwicklung einer Inguinalhernie: Hernienrepair.

- Sekundärer Repositionsverlust: Je nach Ausmaß des Repositionsverlusts in den ersten 10 Tagen erneute Osteosynthese, ansonsten Abwarten der Spontanheilung.

- Sekundäre Koxarthrose: Hüftprothese frühestens nach 3 Monaten.

\section{Ergebnisse}

Von Juni 1997 bis August 2006 wurden insgesamt 17 Patienten mit einer Azetabulum-T-Fraktur chirurgisch versorgt und im Verlauf retrospektiv analysiert (Tabelle 1). Zehn Patienten (59\%) wurden via chirurgische Hüftluxation (Abbildung 30), zwei Patienten $(12 \%)$ über einen Stoppa-Zugang und fünf Patienten (29\%) über einen kombinierten (Abbildung 31) oder alternativen Zugang (klassischer ilioinguinaler $\mathrm{Zu}$ - gang) operiert. Das Patientenkollektiv umfasst acht Frauen und neun Männer mit einem mittleren Alter von 48,8 \pm 20,5 Jahren (19-83 Jahre). Der Traumamechanismus war in der Mehrzahl der Fälle ein Verkehrsunfall ( $\mathrm{n}=8[47 \%])$, seltener ein Sturz aus großer Höhe $(n=3[18 \%])$, ein Skiunfall $(n=3[18 \%])$, ein Niederenergietrauma $(n=1[6 \%])$ oder unbekannt $(n=2$ [11\%]). In drei Fällen (18\%) war zudem eine traumatische, posteriore Hüftluxation vorhanden, welche innerhalb von $6 \mathrm{~h}$ postakzidentell geschlossen reponiert wurde.

Die mittlere Operationszeit betrug 3,3 $\pm 1,7 \mathrm{~h}(1,5-7$ h) für Osteosynthesen mit chirurgischer Hüftluxation, 4,2 $\pm 1,0 \mathrm{~h}(3-5 \mathrm{~h})$ für einen Stoppa-Zugang und 6,4 $\pm 3,1$ h (3,5-10 h) für einen kombinierten oder alternativen Zugang. In fünf Fällen (29\%) wurde im Rahmen der Erstoperation durch eine chirurgische Hüftluxation direkt nach der Osteosynthese eine primäre Hüfttotalprothese implantiert, da der intraartikuläre Schaden derart ausgeprägt war, dass ein gelenkerhaltendes Verfahren wenig erfolgversprechend gewesen wäre. 

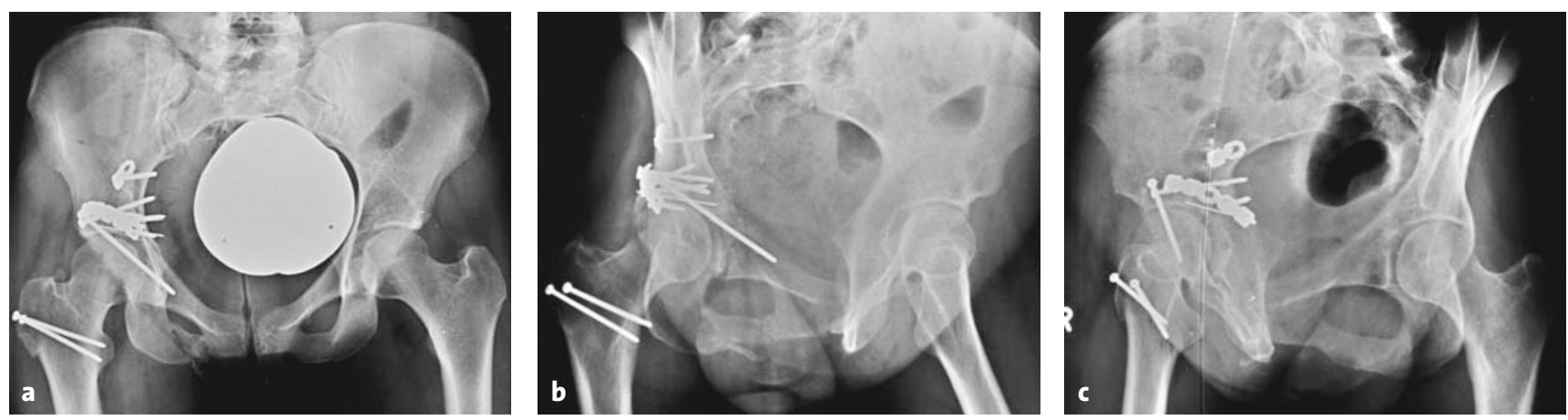

\section{Abbildungen $30 a$ bis $30 e$}

a-c) Postoperative Bilder der 33-jährigen Patientin aus Abbildung 2. Mittels einer chirurgischen Hüftluxation wurde eine anatomische Reposition erreicht. Die etwas überstehenden Schrauben spielten für das klinische Ergebnis keine Rolle. d) Die intraoperative Ansicht des Femurkopfes zeigt deutliche sekundäre Schäden des Gelenkknorpels.
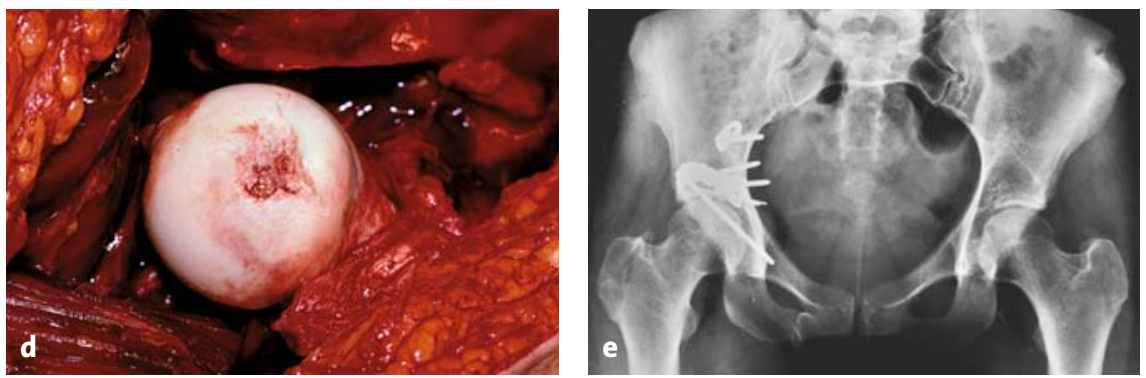

e) In der Verlaufskontrolle 3 Jahre post-

operativ stationäre Gelenkverhältnisse ohne Zeichen einer sekundären Koxarthrose oder Femurkopfnekrose.
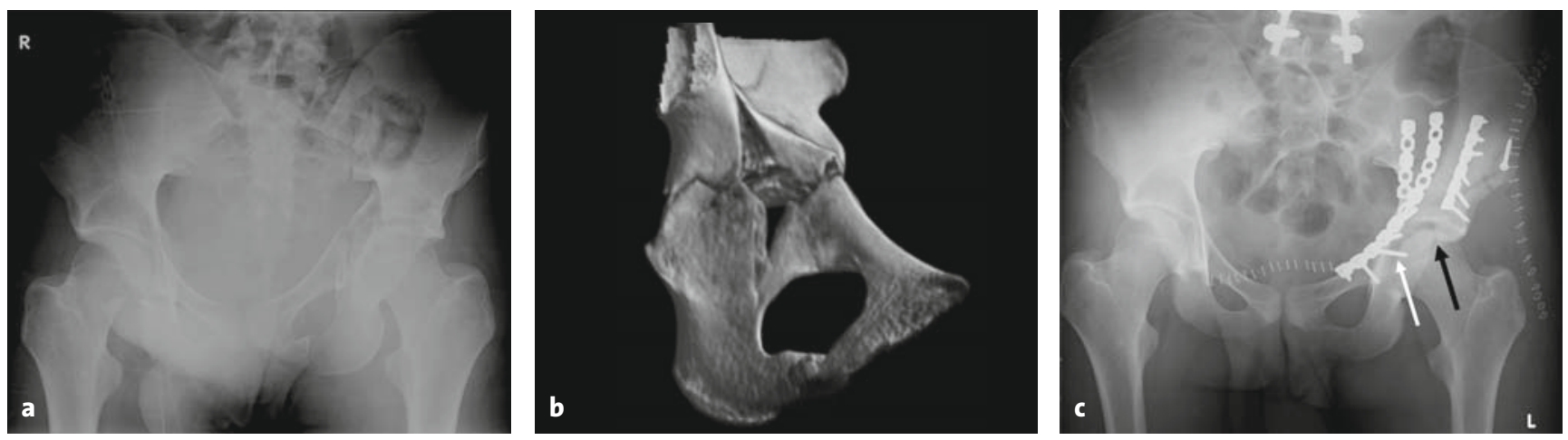

\section{Abbildungen 31a bis $31 e$}

a, b) 19,9-jähriger polytraumatisierter $\mathrm{Pa}$ tient mit T-Fraktur des Azetabulums mit Ausläufer in den vorderen Pfeiler.

c) Via Stoppa-Zugang primäre Fixation des vorderen Pfeilers. Hierunter zeigen sich im postoperativen Bild eine mögliche intraartikuläre Schraubenlage (weißer Pfeil) und eine nichtanatomische Reposition mit einem verbleibenden intraartikulären Frakturspalt (schwarzer Pfeil).

d) Das postoperative Computertomogramm zeigt zudem eine Gelenkimpak-
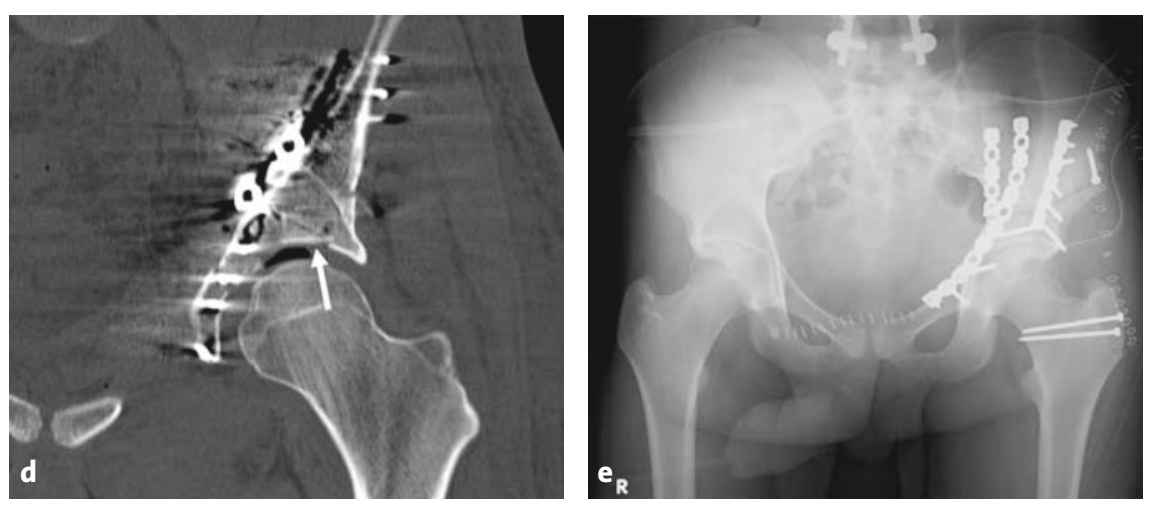
tion.

e) Anatomische Reposition nach chirurgischer Hüftluxation mit Anhebung des impaktierten Fragments, Visualisierung der intraartikulären Schraubenlage und Komplettierung der Osteosynthese. 
In der Regel wurden bei den Patienten postoperativ zur Repositionskontrolle eine anteroposteriore Beckenübersichtsaufnahme sowie beide Letournel-Aufnahmen angefertigt. Die Reposition wurde nach Matta [4] klassifiziert. Bei Patienten mit einer chirurgischen Hüftluxation wurde zudem die direkte intraoperative Visualisierung zur Objektivierung der Gelenkreposition verwendet (Abbildung 30). Insgesamt konnte in zehn der zwölf Osteosynthesen (ohne Primärprothese) eine anatomische Reposition erreicht werden. In zwei Fällen wurde eine zufriedenstellende Reposition konstatiert.

Intraoperativ zeigten sich keine relevanten chirurgischen Komplikationen. Es kam bei keinem Patienten $\mathrm{zu}$ einem iatrogenen Schaden des Nervus ischiadicus oder des Nervus obturatorius. Im postoperativen Verlauf zeigten sich insgesamt bei drei Patienten relevante Komplikationen: eine verzögerte Trochanterheilung ohne Notwendigkeit eines Zweiteingriffs, heterotope Ossifikationen Grad II-III nach Brooker bei einem Patienten sowie ein sekundärer Repositionsverlust.

Alle bis auf fünf Patienten, die entweder verstorben $(n=1)$ oder unbekannt verzogen $(n=4)$ waren, wurden im Rahmen unsere Polikliniksprechstunde klinisch und radiologisch nachkontrolliert. Der mittlere Nachuntersuchungszeitraum betrug 3,2 $\pm 2,3$ Jahre (0,4-7,4 Jahre). Im Rahmen der Verlaufskontrolle wurden routinemäBig eine anteroposteriore Beckenübersicht und eine axiale Aufnahme des Hüftgelenks angefertigt. Bei zwei Patienten musste im Verlauf das Gelenk aufgrund einer sekundären Koxarthrose endoprothetisch versorgt werden (8 Monate und 2,3 Jahre nach der Erstoperation). Im Verlauf wurden keine avaskulären Nekrosen des Femurkopfes detektiert.

Aufgrund der eher geringen Prävalenz von T-Frakturen gibt es derzeit noch keine größeren Studien über
Azetabulum-T-Frakturen, welche über eine chirurgische Hüftluxation und/oder einen Stoppa-Zugang angegangen worden sind. Die chirurgische Hüftluxation sowie der Stoppa-Zugang sind jedoch vielversprechende Erweiterungen/Abänderungen der klassischen Zugänge zur Behandlung von Azetabulumfrakturen mit geringer Morbidität.

\section{Literatur}

1. Cole JD, Bolhofner BR. Acetabular fracture fixation via a modified Stoppa limited intrapelvic approach. Description of operative technique and preliminary treatment results. Clin Orthop 1994;305:112-23.

2. Ganz R, Gill TJ, Gautier E, et al. Surgical dislocation of the adult hip. J Bone Joint Surg Br 2001;83:1119-24.

3. Hong HX, Pan ZJ, Chen X, et al. An anatomical study of corona mortis and its clinical significance. Chin J Traumatol 2004;7:165-9.

4. Matta JM. Fractures of the acetabulum: accuracy of reduction and clinical results in patients managed operatively within three weeks after the injury. J Bone Joint Surg Am 1996;78:1632-45.

5. Okcu G, Erkan S, Yercan HS, et al. The incidence and location of corona mortis: a study on 75 cadavers. Acta Orthop Scand 2004;75:53-5.

6. Siebenrock KA, Gautier E, Woo AK, et al. Surgical dislocation of the femoral head for joint debridement and accurate reduction of fractures of the acetabulum. J Orthop Trauma 2002;16:543-52.

7. Siebenrock KA, Gautier E, Ziran BH, et al. Trochanteric flip osteotomy for cranial extension and muscle protection in acetabular fracture fixation using a Kocher-Langenbeck approach. J Orthop Trauma 1998;12:387-91.

\author{
Korrespondenzanschrift \\ Dr. Moritz Tannast \\ Klinik für Orthopädische Chirurgie \\ Inselspital \\ Universität Bern \\ Freiburgstraße \\ 3010 Bern \\ Schweiz \\ Telefon (+41/31) 632-2222, Fax -3600 \\ E-Mail:moritz.tannast@insel.ch
}

